University of Nebraska - Lincoln

DigitalCommons@University of Nebraska - Lincoln

March 1999

\title{
Mirroring behavior of partial photodetachment and photoionization cross sections in the neighborhood of a resonance
}

Chien-Nan Liu

University of Nebraska - Lincoln

Anthony F. Starace

University of Nebraska-Lincoln, astarace1@unl.edu

Follow this and additional works at: https://digitalcommons.unl.edu/physicsstarace

Part of the Physics Commons

Liu, Chien-Nan and Starace, Anthony F., "Mirroring behavior of partial photodetachment and photoionization cross sections in the neighborhood of a resonance" (1999). Anthony F. Starace Publications. 67.

https://digitalcommons.unl.edu/physicsstarace/67

This Article is brought to you for free and open access by the Research Papers in Physics and Astronomy at DigitalCommons@University of Nebraska - Lincoln. It has been accepted for inclusion in Anthony F. Starace Publications by an authorized administrator of DigitalCommons@University of Nebraska - Lincoln. 


\title{
Mirroring behavior of partial photodetachment and photoionization cross sections in the neighborhood of a resonance
}

\author{
Chien-Nan Liu and Anthony F. Starace \\ Department of Physics and Astronomy, The University of Nebraska, Lincoln, Nebraska 68588-0111
}

(Received 13 October 1998)

\begin{abstract}
Partial photodetachment and photoionization cross sections corresponding to highly excited residual atoms or ions are shown analytically to mirror one another in the neighborhood of a resonance. More precisely, any two groupings of partial cross sections are shown here to have components whose variations with energy near a resonance are equal in magnitude and opposite in direction. This work extends an analysis of Starace [Phys. Rev. A 16, 231 (1977)] for the behavior of partial cross sections near a resonance to the case when the $\rho^{2}$ parameter of Fano and Cooper [Phys. Rev. 137, A1364 (1965)] tends to zero. [S1050-2947(99)50403-4]
\end{abstract}

PACS number(s): $32.80 . \mathrm{Dz}, 32.80 . \mathrm{Fb}, 32.80 . \mathrm{Gc}$

In the early days of vacuum UV spectroscopy, the observation of mirrorlike behavior in partial cross sections in the neighborhood of a resonance was an occasional curiosity. For example, Samson and Cairns [1], in their measurement of the partial cross sections for photoionization of the $5 p$ subshell of $\mathrm{Xe}$ [i.e., $\mathrm{Xe} 5 p^{6}+\gamma \rightarrow \mathrm{Xe}^{+} 5 p^{5}\left({ }^{2} P_{J}\right)+e^{-}$, where $J=3 / 2$ or $1 / 2]$, note that " $\ldots$ at the $543 \AA$ resonance the $\sigma_{3 / 2}$ cross section decreased while the $\sigma_{1 / 2}$ cross sections increased by almost the same magnitude. The net result was that the two large resonances [i.e., in each partial cross section] practically annulled each other such that only a weak resonance could be observed in the total cross section curve.' At the present time, more than 30 years later, advances in computer power on the one hand and in the intensity and resolution of experimental light sources as well as in both target preparation and interaction product detection techniques on the other hand have permitted theorists and experimentalists to study partial cross sections for photodetachment and photoionization accompanied by high levels of excitation of the residual atom or ion. In these recent studies, the observation of mirroring behavior among different partial cross sections is common; see, e.g., Refs. [2] and [3].

We present here an analytic proof that such mirroring behavior among partial photodetachment or photoionization cross sections is to be expected in the neighborhood of resonances when these partial cross sections correspond to high levels of excitation of the residual atom or ion. Our analysis is based on the work of one of us [4] concerning the general form of electric dipole transition amplitudes in the vicinity of an isolated resonance. This work [4] generalized the earlier analyses of Fano and Cooper [5-7] on the behavior of total cross sections in the vicinity of an isolated resonance. Our analysis is based on a key feature of partial photodetachment and photoionization cross sections for high excitations of the residual atom or ion. That is, the doubly-excited states that produce the most dramatic effects on these highly excited partial cross sections generally have only a small effect on the total cross section, which has the following general form [7]:

$$
\sigma_{T O T}=\sigma_{T O T}^{0}\left(\rho^{2} \frac{(q+\epsilon)^{2}}{1+\epsilon^{2}}+1-\rho^{2}\right)
$$

In Eq. (1) $\sigma_{T O T}^{0}$ is the total cross section in the absence of the resonance and $\rho^{2}$ is the maximum fractional depth of the minimum of the total cross section in the vicinity of a resonance [7]. Thus, the simplest way to describe mathematically the observed features of resonance behavior on total cross sections in recent calculations and measurements (for photodetachment or photoionization accompanied by high excitation of the residual atom or ion) is to state that the Fano and Cooper parameter $\rho^{2}[6,7]$ is small. Therefore, in order to explore the origin of the observed mirroring behavior in the corresponding partial cross sections, we examine here the effect of an isolated resonance on partial cross sections for the case in which $\rho^{2} \rightarrow 0$.

Our analysis is based upon a few key formulas from Ref. [4]. The main one is for the ratio of two partial cross sections in the vicinity of an isolated resonance, given by Eq. (28) of Ref. [4]. We write that result here in the form of the individual partial cross sections, denoted by $P$ and $Q$,

$$
\begin{aligned}
\sigma_{P}= & \frac{\sigma_{P}^{0}}{1+\epsilon^{2}}\left\{\epsilon^{2}+2 \epsilon\left(q \operatorname{Re}\langle\alpha\rangle_{P}-\operatorname{Im}\langle\alpha\rangle_{P}\right)\right. \\
& \left.+\left[1-2 q \operatorname{Im}\langle\alpha\rangle_{P}-2 \operatorname{Re}\langle\alpha\rangle_{P}+\left(q^{2}+1\right)\left\langle|\alpha|^{2}\right\rangle_{P}\right]\right\} .
\end{aligned}
$$

$\sigma_{Q}$ has an identical form to that in Eq. (2), but with $P$ replaced by $Q$. In Eq. (2), $\sigma_{P}^{0}$ is the partial cross section in the absence of the resonance, $\epsilon$ and $q$ are the well-known Fano profile variables [5], which come from his analysis of the effects of the resonance on the total cross section, $\sigma_{T O T}$ $=\sigma_{P}+\sigma_{Q}$, and $\langle\alpha\rangle_{P}$ and $\left\langle|\alpha|^{2}\right\rangle_{P}$ are parameters introduced by Starace [4] to describe the effects of a resonance on a partial cross section. Now, as shown in Eqs. (36) and (40) of Ref. [4], the $\alpha$ parameters for the $P$ and $Q$ partial cross sections are related to one another by means of the correlation index $\rho^{2}$ of Fano and Cooper [6,7]:

$$
\begin{gathered}
\sigma_{P}^{0}\left\langle|\alpha|^{2}\right\rangle_{P}+\sigma_{Q}^{0}\left\langle|\alpha|^{2}\right\rangle_{Q}=\rho^{2} \sigma_{T O T}^{0}, \\
\sigma_{P}^{0}\langle\alpha\rangle_{P}+\sigma_{Q}^{0}\langle\alpha\rangle_{Q}=\rho^{2} \sigma_{T O T}^{0} .
\end{gathered}
$$

Before proceeding further, we note that, in photodetachment or photoionization accompanied by excitation of the 
atom or ion, there are in general many more than just two partial cross sections. Nevertheless, our analysis is applicable since one may always group however many partial cross sections there are into two groups. The more partial cross sections there are, the more ways there are of forming two groups of partial cross sections, denoted by $P$ and $Q$. Our results below imply that any such grouping will exhibit mirroring behavior in the vicinity of an isolated resonance.

The squared correlation index $\rho^{2}$ measures the fraction of the total cross section that interacts with the resonance. The theoretical expression of $\rho^{2}$ is given in Eq. (A2). Fano and Cooper have interpreted this expression for $\rho^{2}$ as the square of an overlap of two continuum states, one of which is generated by autoionization of the doubly-excited state $\phi$ and the other of which is generated by direct photoabsorption from the initial state $\psi_{0}$ [7]. Therefore, $\rho^{2}$ indicates the strength of the interaction between the autoionization state and the continuum. For doubly-excited states near highly excited thresholds, the overlap with the continuum states associated with lower levels of excitation of the residual atom or ion is small, and thus $\rho^{2}$ is small. We therefore examine Eq. (2) in the limit when $\rho^{2} \rightarrow 0$. Equation (3) combined with the definitions of $\langle\alpha\rangle_{P}$ and $\left\langle|\alpha|^{2}\right\rangle_{P}$ in Eqs. (30) and (31) of Ref. [4] imply that $\left\langle|\alpha|^{2}\right\rangle_{P}=\left\langle|\alpha|^{2}\right\rangle_{Q}=0$ for $\rho^{2} \rightarrow 0$, and that $\langle\alpha\rangle_{P}=\langle\alpha\rangle_{Q}=0$. Thus Eq. (2) seems to take the limit $\sigma_{P}$ $\rightarrow \sigma_{P}^{0}$ when $\rho^{2} \rightarrow 0$. However, this simple analysis is incorrect since it ignores the behavior of $q$ as $\rho^{2} \rightarrow 0$.

As shown in the Appendix, $q \rho^{2} \rightarrow 0$ in the limit when $\rho^{2} \rightarrow 0$, but $q^{2} \rho^{2}$ remains finite. In fact, as implied by Eq. (1), $q^{2} \rho^{2}$ measures the fractional rise of the cross section in the vicinity of a resonance above that which would be the case in the absence of the resonance [7]. Taking the behaviors of $q \rho^{2}$ and $q^{2} \rho^{2}$ into account, Eqs. (3) and (4) imply that, as $\rho^{2} \rightarrow 0$, we have

$$
\begin{gathered}
\lim _{\rho \rightarrow 0} \sigma_{Q}^{0} q^{2}\left\langle|\alpha|^{2}\right\rangle_{Q}=\rho^{2} q^{2} \sigma_{T O T}^{0}-\sigma_{P}^{0} q^{2}\left\langle|\alpha|^{2}\right\rangle_{P}, \\
\lim _{\rho \rightarrow 0} q \sigma_{Q}^{0}\langle\alpha\rangle_{Q}=-q \sigma_{P}^{0}\langle\alpha\rangle_{P} .
\end{gathered}
$$

In the limit when $\rho^{2} \rightarrow 0$, the partial cross sections can now be written as

$$
\begin{aligned}
\lim _{\rho \rightarrow 0} \sigma_{P}= & \sigma_{P}^{0}+\frac{\sigma_{P}^{0}}{1+\epsilon^{2}}\left\{2 q\left[\epsilon \operatorname{Re}\langle\alpha\rangle_{P}-\operatorname{Im}\langle\alpha\rangle_{P}\right]\right. \\
& \left.+q^{2}\left\langle|\alpha|^{2}\right\rangle_{P}\right\}, \\
\lim _{\rho \rightarrow 0} \sigma_{Q}= & \sigma_{Q}^{0}+\frac{\sigma_{P}^{0}}{1+\epsilon^{2}}\left\{-2 q\left[\epsilon \operatorname{Re}\langle\alpha\rangle_{P}-\operatorname{Im}\langle\alpha\rangle_{P}\right]\right. \\
& \left.-q^{2}\left\langle|\alpha|^{2}\right\rangle_{P}+\rho^{2} q^{2}\left(\frac{\sigma_{T O T}}{\sigma_{P}^{0}}\right)\right\},
\end{aligned}
$$

where in Eq. (8) we have used Eqs. (5) and (6) and have set terms involving $\alpha$ parameters that are not multiplied by $q$ or $q^{2}$ equal to zero. Summing Eqs. (7) and (8), we find

$$
\lim _{\rho \rightarrow 0} \sigma_{T O T}=\lim _{\rho \rightarrow 0}\left(\sigma_{P}+\sigma_{Q}\right)=\sigma_{T O T}^{0}\left\{1+\frac{\rho^{2} q^{2}}{1+\epsilon^{2}}\right\} .
$$

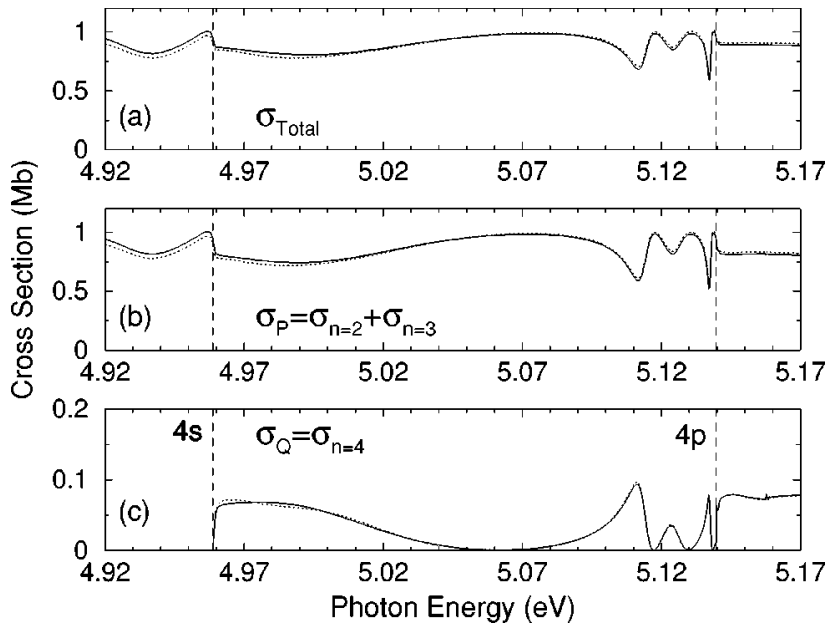

FIG. 1. Cross sections for the process $\mathrm{Li}^{-}+\gamma \rightarrow \mathrm{Li}(n)+e^{-}$calculated using the eigenchannel $R$-matrix approach of Refs. [2,3]. Solid (dotted) curves indicate dipole velocity (length) results. (a) Total cross section. (b) Sum of the partial cross sections for $n=2$ and $n=3$. (c) Partial cross section for $n=4$.

We point out here certain features of the last three equations. First, note that, unlike Eq. (1), Eq. (9) does not have an interference term proportional to $\epsilon /\left(1+\epsilon^{2}\right)$. The term proportional to $\rho^{2} q^{2}$ represents a Lorentzian autoionization profile. Nevertheless, although there are no interference effects in the total cross section, Eqs. (7) and (8) indicate interference behavior in the partial cross sections. Second, Eq. (9) implies that the total cross section cannot be zero, but Eqs. (7) and (8) allow the possibility that the partial cross sections may have a zero minimum. Third, and most important, we see that the energy-dependent parts of $\sigma_{P}$ in Eq. (7) and $\sigma_{Q}$ in Eq. (8) are equal in magnitude and opposite in sign except for the term $\rho^{2} q^{2} \sigma_{T O T} /\left(1+\epsilon^{2}\right)$ in Eq. (8). Thus, we have shown analytically that the two cross sections mirror each other's behavior. This mirroring will be more pronounced the smaller the term involving $\rho^{2} q^{2}$ in Eq. (8) is, or, alternatively, the smaller the effect of the resonance on the total cross section is.

In Fig. 1, we illustrate the mirroring behavior that Eqs. (7) and (8) can interpret for the specific case of $\mathrm{Li}^{-}$photodetachment for photon energies between the $\operatorname{Li}(4 s)$ and $\operatorname{Li}(4 p)$ thresholds. These results are obtained using the same eigenchannel $R$-matrix methods described in Refs. [2,3]. If one indicates by $\sigma_{n l}$ the partial cross section for the process,

$$
\mathrm{Li}^{-}+\gamma \rightarrow \mathrm{Li}(n l)+e^{-}
$$

then the total cross section is given by $\sigma_{\text {Total }}=\Sigma_{n l} \sigma_{n l}$, where the sum extends over all excited atomic states of $\mathrm{Li}$ allowed in the photon-energy range shown, i.e., $2 s \leqslant n l$ $\leqslant 4 s$. For simplicity of notation, we consider the partial cross sections $\sigma_{n}=\Sigma_{l} \sigma_{n l}$, where the sum over $l$ is over all allowed excitations $\operatorname{Li}(n l)$ below the $\operatorname{Li}(4 p)$ threshold. In the $P, Q$ notation of this paper, we divide the total cross section into two groups,

$$
\begin{gathered}
\sigma_{P}=\sigma_{n=2}+\sigma_{n=3}, \\
\sigma_{Q}=\sigma_{n=4} .
\end{gathered}
$$


As shown in Fig. 1(a), $\sigma_{\text {Total }}=\sigma_{P}+\sigma_{Q}$ shows significant resonance structure just below the $\operatorname{Li}(4 p)$ threshold. There are actually four resonances in the energy region between the $\mathrm{Li}(4 s)$ and $\operatorname{Li}(4 p)$ thresholds, which while not isolated are clearly well separated. (Our calculations locate these resonances at $\hbar \omega=5.024,5.112,5.119$, and $5.138 \mathrm{eV}$.) Also, while $\rho^{2}$ is clearly far from zero for each of these resonances, it is nevertheless small, i.e., far less than unity. Figure 1(b) shows that $\sigma_{P}$ is almost equal in magnitude to $\sigma_{\text {Total }}$. However, the resonance window features just below the $\operatorname{Li}(4 p)$ threshold are more pronounced, i.e., they have lower minima than in $\sigma_{\text {Total }}$. Even the broad cross-section trough in $\sigma_{P}$ in the vicinity of the $\hbar \omega=5.024 \mathrm{eV}$ resonance is lower than that in $\sigma_{\text {Total }}$. In fact, as shown by a comparison of Figs. 1(b) and 1(c), the lower window minima in $\sigma_{P}$ are precisely mirrored by peak structures in $\sigma_{Q}$ and the trough feature in $\sigma_{P}$ is mirrored by a shoulder feature in $\sigma_{Q}$. Finally we note the near zero minima in $\sigma_{Q}$. All of these behaviors are fully in accord with our analytic predictions in Eqs. (7) and (8).

This work has been supported in part by the U.S. Department of Energy, Office of Basic Energy Sciences, under Grant No. DE-FG03-96ER14646.

\section{APPENDIX}

Equations (22) and (32) of Ref. [4] define the $q$ and $\rho^{2}$ parameters of Fano and Cooper [5-7] as follows:

$$
\begin{gathered}
\left\langle\psi_{0}|\mathbf{r}| \Phi\right\rangle=\pi q \sum_{i=1}^{N}\left\langle\psi_{0}|\mathbf{r}| i E\right\rangle\langle i E|V| \phi\rangle, \\
\left(\sum_{i=1}^{N}\left\langle\psi_{0}|\mathbf{r}| i E\right\rangle\langle i E|V| \phi\rangle\right)^{2}=\rho^{2} \frac{\Gamma(E)}{2 \pi} \sum_{\mu=1}^{N}\left|\left\langle\psi_{0}|\mathbf{r}| \mu E_{-}\right\rangle\right|^{2} .
\end{gathered}
$$

In these equations, $\left\langle\psi_{0}|\mathbf{r}| \Phi\right\rangle$ is the electric dipole transition matrix element from the initial state $\psi_{0}$ to the modified resonance state $\Phi$ (i.e., modified by its interaction with the continuum), $\left\langle\psi_{0}|\mathbf{r}| i E\right\rangle$ is the electric dipole transition matrix element from the initial state $\psi_{0}$ to the $i$ th continuum channel with total energy $E,\langle i E|V| \phi\rangle$ describes the autoionization matrix element between the bare resonance state $\phi$ and the continuum channel $i$ at energy $E$, and $\Gamma(E)$ is the width of the resonance. See Refs. [4-7] for a more detailed description. Normally, both $\Gamma(E)$ and $\sum_{\mu=1}^{N}\left|\left\langle\psi_{0}|\mathbf{r}| \mu E_{-}\right\rangle\right|^{2}$ are finite, so that $\rho^{2} \rightarrow 0$ implies $\sum_{i=1}^{N}\left\langle\psi_{0}|\mathbf{r}| i E\right\rangle\langle i E|V| \phi\rangle \rightarrow 0$. Therefore, assuming $\left\langle\psi_{0}|\mathbf{r}| \Phi\right\rangle$ is not equal to $0, q$ in Eq. (A1) is not well defined. Since Eq. (2) for the partial cross sections $\sigma_{P}$ or $\sigma_{Q}$ has terms involving, respectively, $q\langle\alpha\rangle_{P}$ or $q\langle\alpha\rangle_{Q}$ and $q^{2}\left\langle|\alpha|^{2}\right\rangle_{P}$ or $q^{2}\left\langle|\alpha|^{2}\right\rangle_{Q}$, we must consider their behaviors as $\rho \rightarrow 0$. Consider first the terms involving $q^{2}\left\langle|\alpha|^{2}\right\rangle$. Multiplying Eq. (3) by $q^{2}$ and substituting Eqs. (A1) and (A2) for $q$ and for $\rho^{2}$, we have that

$$
\begin{aligned}
& q^{2}\left(\sigma_{P}^{0}\left\langle|\alpha|^{2}\right\rangle_{P}+\sigma_{Q}^{0}\left\langle|\alpha|^{2}\right\rangle_{Q}\right) \\
& \quad=q^{2} \rho^{2} \sigma_{T O T}^{0} \\
& \quad=\left(\frac{2}{\pi \Gamma(E)}\right)\left(\frac{\left|\left\langle\psi_{0}|\mathbf{r}| \Phi\right\rangle\right|^{2}}{\sum_{\mu=1}^{N}\left|\left\langle\psi_{0}|\mathbf{r}| \mu E_{-}\right\rangle_{0}\right|^{2}}\right) \sigma_{T O T}^{0} \\
& \quad \geqslant 0 .
\end{aligned}
$$

Similarly, multiplying Eq. (4) by $q$ and substituting Eqs. (A1) and (A2), we find that

$$
\begin{aligned}
q\left(\sigma_{P}^{0}\langle\alpha\rangle_{P}+\sigma_{Q}^{0}\langle\alpha\rangle_{Q}\right) & =q \rho^{2} \sigma_{T O T}^{0} \\
& \propto \sum_{i=1}^{N}\left\langle\psi_{0}|\mathbf{r}| i E\right\rangle\langle i E|V| \phi\rangle \rightarrow 0 .
\end{aligned}
$$

Equations (A3) and (A4) prove the $\rho \rightarrow 0$ limits given in Eqs. (5) and (6) above.
[1] J. A. R. Samson and R. B. Cairns, Phys. Rev. 173, 80 (1968). The quoted text appears on p. 84.

[2] C. Pan, A. F. Starace, and C. H. Greene, Phys. Rev. A 53, 840 (1996). Compare the partial cross section for photodetachment of $\mathrm{Li}^{-}$to the $\mathrm{Li}(3 s)$ state in Fig. 6(b) with the partial cross section to the $\mathrm{Li}(4 s)$ state in Fig. 6(c) for photon energies below the $\operatorname{Li}(4 p)$ threshold. These predictions have been confirmed experimentally by G. Haeffler, I. Yu. Kiyan, D. Hanstorp, and D. J. Pegg, ibid. 57, 2216 (1998).
[3] C. N. Liu and A. F. Starace, Phys. Rev. A 58, 4997 (1998). Compare the partial cross sections for the process $\mathrm{Li}^{-}+\gamma \rightarrow \operatorname{Li}(n l)+e^{-}$for $n l=4 s, 4 p, 4 d, 4 f$, and $5 s$ in Fig. 5.

[4] A. F. Starace, Phys. Rev. A 16, 231 (1977).

[5] U. Fano, Phys. Rev. 124, 1866 (1961).

[6] U. Fano and J. W. Cooper, Phys. Rev. 137, A1364 (1965).

[7] U. Fano and J. W. Cooper, Rev. Mod. Phys. 40, 441 (1968), Sec. 8.1. 